\title{
Variable Particle Positioning in Transitive Phrasal Verbs
}

\author{
By Manuela C. de Oliveira*
}

\begin{abstract}
The present work investigates the English transitive phrasal verb construction (TPV) and the reasons for its variable particle placement. Particles can be placed continuously or discontinuously in relation to the construction verb, e.g. "turn off the computer" and "turn the computer off", respectively. Several studies point out that noun phrase (NP) length (Hawkings 1994, Gorlach 2004, Lohse et al. 2004, Quirk et al. 1985) and idiomaticity (Gries 2001, Gorlach 2004) motivate particle placement. A study of particles "away", "off" and "out" in TPVs is developed in favour of an account that considers specific constructions as chunks due to repetition. The hypothesis supported in this work associates loss of analysability and gain of entrenchment between verb and particle with high token frequency. Thus, a TPV becomes a sequence with verb + particle packaged together and accessed as a unit in consequence of its frequent use (Bybee 2010). Written discourse data collected from BNC (British National Corpus) show a significant preference for the continuous order in spite of constructions' variable levels of compositionality and idiomaticity. In relation to particles "away", "off" and "out", there is a difference in frequency and range of verbs used with each particle: while "away" is part of only $8 \%$ of the data and "off", 26\%, the particle "out" is present in 66\% of all TPVs, which makes "out" both the most recurrent particle in the sample and the one with highest type frequency: 96 different verbs were part of "out" constructions. Results also demonstrate that some verbs are used in both continuous and discontinuous order, whereas others are restricted to one construction only: "take" joins all three particles and in both orders, while "carry" is always immediate to "out". NPs that follow "carry out" all bear semantic resemblance, indicating they form sequential and indivisible units which seem to be motivated by the NP characteristics.
\end{abstract}

Keywords: Compositionality, Construction, Particles, Phrasal verbs, Transitive

\section{Introduction}

A phrasal verb is a construction formed by verb and particle, which together convey an idea often different from the one transmitted by the verb alone. The lexical verb take, for example, can express one meaning, while it has a different meaning when part of a combination with the particle up:

1. The town took the land by eminent domain ${ }^{1}$.

2. Let's take up each problem one at a time.

In the examples above, take means to seize with authority or legal right while take up means to deal with a situation. The latter will be referred to as an example of a Transitive Phrasal Verb (TPV) and it will be the focus of this paper.

TPVs may vary in terms of idiomaticity, considering that some constructions are closer to an idiomatic pole and others, to a more literal pole. Observe examples (3) and (4) to tackle this matter:

\footnotetext{
${ }^{*} \mathrm{PhD}$ Student, Federal University of Rio de Janeiro - UFRJ, Brazil.

1 "Take". TheFreeDcitionary.com. Retrieved from goo.gl/qJ7Fh. [Accessed: 4 January 2016] 
3. Mary needs to take back the shirt she lent you.

4. I gave up smoking ${ }^{2}$.

In (3) the meaning of take + back is transparent and it can be retrieved through the sum of the meanings of take and back alone. On the other hand, the meaning of give up (4) is not retrieved through the understanding of verb and particle independently. One must be aware that the combination of give and up means to stop doing something.

Depending on the level of idiomaticity (Gorlach 2004, Quirk et al. 1985) particles are allowed to be placed adjacent to the verb or to the NP, thus combinations can be referred to as continuous (V P NP) or discontinuous (V NP P). Example (3) illustrates a continuous combination: take+ back+ the shirt and the discontinuous form could be represented by take+ the shirt + back.

This work investigates the use of TPVs in written discourse in regards to particles away, off and out collected from the British National Corpus (BNC). When the particle is adjacent to the verb, the construction will be referred to as continuous, and discontinuous if NP is separated by verb and particle (Haddican and Johnson 2012). The hypothesis that has been put forward in this paper is that the frequency to which some TPVs are used lead them into forming chunks, thus TPVs lose analysability and compositionality as a result of frequent repetition (Bybee 2010). Therefore, verb and particle are not always selected individually to form a construction whose interpretation comes from the sum of each part. In fact, they are accessed as indivisible sequential units conventionalized in the system. An overview of the constructs exposes a significant preference for the continuous order (V P NP) in spite of general motivations such as idiomaticity and/or NP length (Hawkings 1994, Gries 2001).

\section{Multi-word Verbs}

English verbs may be paired with particles in various types of combinations. In Biber et al. (2010), multi-word combinations such as verbparticle ones are split into two categories: intransitive or transitive. Intransitive constructions may be semantically more compositional in meaning, so they are referred to as free combinations (5), but if their meaning is more idiomatic, one should refer to them as phrasal verbs (6):

5. I will come back in a minute.

6. Stop being so childish and grow up.

Example (5) is a free combination because $\mathrm{V}$ and $\mathrm{P}$ express independent ideas. The particle back is used in numerous constructions often relating to the concept of return, as it was used with the verb come in (5). The combination grow $u p$, on the other hand, is formed by items that are dependent on each other, and (6) illustrates a phrasal verb because its interpretation is not depicted from the individual constituents alone, as with free combinations.

\footnotetext{
2 "Give up". TheFreeDcitionary.com. Retrieved from goo.gl/fyvX7b. [Accessed: 7 January 2016]
} 
In addition, Biber et al. (2010) point out that TPVs differ from prepositional verbs because verb and particle in TPVs demand a complement whose position may vary (7), but this variation does not occur with prepositional verbs (8):

7. a. He picked up the phone.

b. He picked the phone up.

8. a. She is thinking about her friends.

b. ${ }^{*}$ She is thinking her friends about.

While the phrasal verb's particle may be adjacent to the verb (7a) or to the NP (7b), prepositional verbs do not allow this movement (8) and, for that reason, the particle is often referred to as preposition. Taking into consideration the definitions established so far, a schema representation is proposed as follows in order to identify where TPVs are located in relation to multi-word verbs. Figure 1 illustrates a linguistic schema for multi-word verbs based on Biber et al.'s (2010) definition $^{3}$ and the network system proposed by Traugott and Trousdale (2013).

Figure 1. Schematic Representation of Multi-Word Verbs

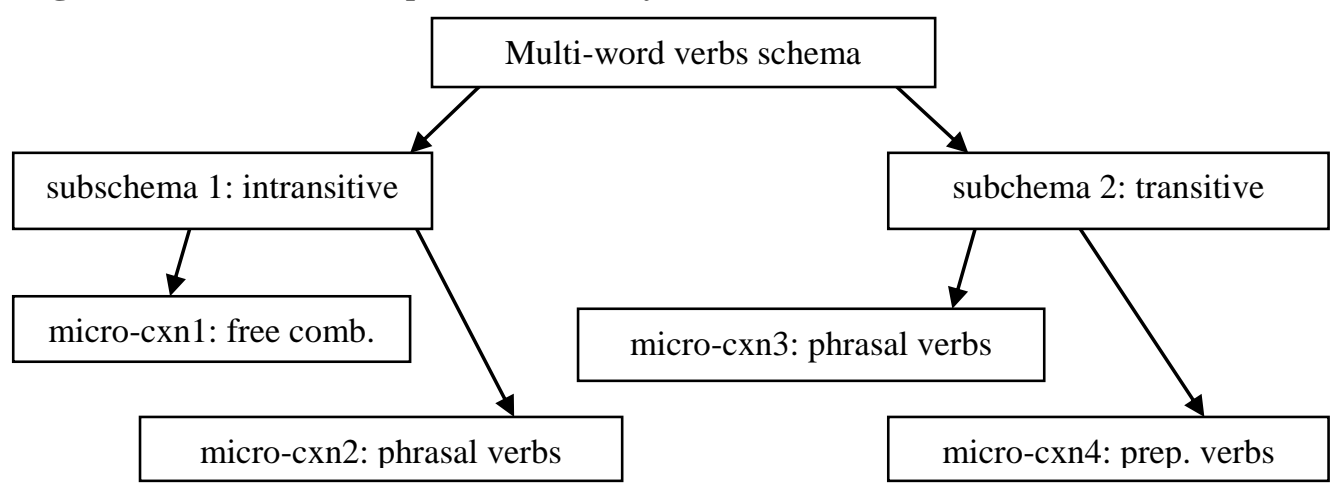

The transitive subschema (2) is formed by two micro-constructions: phrasal verbs and prepositional verbs. Micro-cxn3 has particle variable positioning while micro-cxn4 does not allow such variation.

This paper focuses on micro-construction 3 - as exemplified in (7) - and the motivations for variable particle positioning in this type of construction. The next section argues in favour of an analysis of specific phrasal verbs, in order to observe how levels of analysability and compositionality can interfere in the choice of joint or split orderings by users of English.

\footnotetext{
${ }^{3}$ Phrasal prepositional verbs, such as end up with are regarded by Biber et al. (2010) as one of the transitive constructions. Since their constituents always occur in the same order, they were not considered to be relevant in terms of token frequency for the purpose of this work.
} 


\section{Compositionality and Chunking in TVPs}

In general, phrasal verbs transmit ideas that may or may not be transparent, i.e. predictable from their constituents. If put in a continuum, some constructions of the type are more semantically compositional than others. However, this judgment is gradient since it is not possible to say that one phrasal verb has an entirely idiomatic meaning, whereas another one is completely literal. While some phrasal verbs are easily comprehensible through the meaning of each unit put together, many times one is prevented from fully understanding a construct if one has not learned or experienced it in a previous context. The particle off, for instance, follows a great variety of verbs such as $\log$. If an individual has never heard the phrasal verb log off before, but knows the meaning of $\log$ and off alone, he or she can predict what (9) means:

\section{Come out of Microsoft. Don't forget to tell it to log off the network ${ }^{4}$.}

Because $\log$ off $+\mathrm{NP}$ is highly compositional in terms of its semantics, meaning is detectable from the sum of $\log$ and off. Constituents are independent: the antonym counterpart of (9) is possible through the replacement of off for in/on (e.g. The system is unable to log you in/on). However, it is not always the case that TPV's bear such compositional ideas. Example (10) shows the combination give + $u p+N P$ :

10. The ruling class won't give up its power and wealth voluntarily ${ }^{5}$.

The idea conveyed by give up is only meaningful when both items are used together and continuously. The contrary idea conveyed by the construction give up is not composed by the replacement of particle, but rather through the usage of a new construction, as the following example illustrates:

11. The ruling class won't withstand its power and wealth voluntarily.

While the construction that can express an opposite idea to $\log$ off is $\log \mathrm{in} / \mathrm{on}$, i.e. through particle changing, a contrary idea to give up can only be achieved through a complete change of construction, such as the lexical verb withstand. In other words, give and up have a much stronger semantic link than $\log$ and off.

The considerations made so far serve as evidence to support the claim that "non-compositionality entails that combinations must be stored" (Goldberg 2016: 3 ). As reported by the author, verb-particle combinations ought to have a mental representation in cognition, and the fact that many of them are non-identifiable by their individual parts indicates they are packaged and stored together. Bybee (2010) argues that when two or more words are frequently used together, they may

\footnotetext{
${ }^{4}$ Data cited herein have been extracted from the British National Corpus Online service, managed by Oxford University Computing Services on behalf of the BNC Consortium. All rights in the texts cited are reserved.

5 Data cited herein have been extracted from the British National Corpus Online service, managed by Oxford University Computing Services on behalf of the BNC Consortium. All rights in the texts cited are reserved.
} 
turn into sequential units and tend to become crystallized in a language, i.e. chunks. According to the author, language chunks suffer loss of analysability and compositionality due to their constant use.

The hypothesis for this study is that one order is preferably used, but this choice is particle dependent. In other words, different particles may be more frequent in discontinuous or continuous order. A preference for a specific order expands to other types, being one order a semantic model for the other constructs.

In view of examining word order within the constructions, token and type frequency of the construct, a corpus analysis takes place. Some of the questions I intend to answer are: a) Is there a difference in word ordering linked to specific particles? That is, is there a continuous or discontinuous preference for each particle? b) Do NPs characteristics motivate choice between one variant over another? c) Are there verbs and particles used so often together to the point of being considered chunks? The next section focuses on the approach selected for this paper with the purpose of answering the questions previously mentioned.

\section{Methodology}

According to usage-based theories, as language is used and categorized by users, cognitive representations are built up, making a cognitive representation of a person's experience with language (Bybee and Eddington 2006, Kemmer and Barlow 2000). Hypotheses towards language use can be drawn from experiments, child language acquisition and corpora, among others, for they represent natural language usage. Aiming at analysing particle placement in TPVs and controlling which constructions are more preferred (continuous or discontinuous), an approach based on usage and corpus data was followed.

Data were extracted from the British National Corpus (BNC) so as to verify the behaviour of such constructions. The BNC is a 100 million word synchronic corpus formed by written and spoken samples in 4.049 different texts from the later part of the $20^{\text {th }}$ century. The BNC covers a wide range of sources and it deals with modern British English even though non-British English and foreign language words may occur in the corpus. The spoken English sample is $10 \%$ of the corpus, while $90 \%$ is formed by written samples. The latter includes books (60\%), periodicals, magazines and newspapers (30\%). From the $30 \%$ of periodicals, magazines and newspapers, 250 titles are issues of newspapers.

In this analysis, I have selected a written sample, which covers news with the following topics: arts, commerce, reports, science and social. Taking into account the wide number of particles that join verbs to form phrasal verbs, the list of particles to be verified was narrowed down to three among the most frequent ones (Claridge 2000) in this type of construction: away, off and out. The particle away was used 443 times in 147 different texts from which 78 contained transitive phrasal verb constructions; for the particle off, there were 851 hits in 167 texts from which 262 were part of TPVs; out was used 2.706 times and in 190 different texts from which 600 were TPVs. Table 1 represents the frequency of each particle's use in relation to the sample. 
Table 1. Number of TPVs according to each Particle - Written Sample

\begin{tabular}{|l|c|}
\hline Particle & Frequency \\
\hline Away & $78=8.3 \%$ \\
\hline Off & $262=27.9 \%$ \\
\hline Out & $600=63.8 \%$ \\
\hline Total & $\mathbf{9 4 0}=\mathbf{1 0 0 \%}$ \\
\hline
\end{tabular}

Source: The British National Corpus (BNC).

According to the results, not only is out present in a higher number of tokens, but it is also more frequent than away and off (63.8\%) when it comes to transitive phrasal verbs. Examples of continuous and discontinuous constructions are provided according to the three particles. Letter (a) is continuous and letter (b) is discontinuous; verb and particle are in bold, and NPs are underlined ${ }^{6}$ :

12. a. The biggest task of the Leftist Unity party will be to lure away the working class from the Socialists.

b. The other problem with savings accounts is that inflation eats the investment away.

13. a. Annabel Salmon, Eurotunnel's official spokeswoman, yesterday called off her engagements on the grounds that she has lost her voice.

b. It's the nature of the violence that puts people off.

14. a. Ciba-Geigy, the Swiss chemical and drugs giant, continues to seek out niche markets with a vengeance.

b. Nigel Lawson has now made two attempts to smooth the system out.

Sentences (12) to (14) exemplify each variant and show NPs from one to two words in length. Many of the NPs with two words included possessive pronouns, as in (13a), or articles, as in (12a; 12b) and (14b). When NPs were formed by one constituent, the vast majority were anaphoric pronouns: smooth it out or call her off, for instance. Considering that this type of NP only occurs in the discontinuous order due to the fact that a continuous ordering would be ungrammatical (*smooth out $i t$; *call off her), they do not statistically count in this paper for there is no variable particle positioning in such cases.

In the next section, the results obtained from the BNC corpus in relation to the particles mentioned are provided along with an analysis on the position of the particles and their NPs.

\section{Results}

As previously stated, TPVs containing pronouns were not considered for statistics, due to the fact that there is no variation in particle positioning when they are used. The results for continuous and discontinuous order in relation to each particle are displayed in Table 2.

\footnotetext{
${ }^{6}$ Data cited herein have been extracted from the British National Corpus Online service, managed by Oxford University Computing Services on behalf of the BNC Consortium. All rights in the texts cited are reserved.
} 
Table 2. Particle Placement in TPVs - Written Discourse: News

\begin{tabular}{|l|c|c|c|}
\hline Particle & Continuous & Discontinuous & Frequency \\
\hline Away & $61=89.7 \%$ & $7=10.3 \%$ & $68=7.7 \%$ \\
\hline Off & $217=93.1 \%$ & $16=6.9 \%$ & $233=26.6 \%$ \\
\hline Out & $555=96.3 \%$ & $21=3.64 \%$ & $576=65.7 \%$ \\
\hline Total & & $\mathbf{8 7 7}=\mathbf{1 0 0 \%}$ \\
\hline
\end{tabular}

Source: The British National Corpus (BNC).

From the 78 tokens of particle away in news-written discourse, 10 had pronouns for complements, which makes $7.7 \%$ of transitive phrasal verbs with away. The continuous order was more frequent (89.7\%) than the discontinuous one $(10.3 \%)$. As a matter of fact, this result is related to all three particles, since off is $93.1 \%$ of the times continuous to the verb and out, $96.7 \%$ were part of continuous constructions. In conclusion, there is a large preference for away, off and out to be adjacent to their verbs in the written discourse sample used in this work.

It has been suggested before that variability concerning particle positioning in TPVs has to do with factors that include the amount of linguistic material functioning as the complement of verb + particle. Hawkings (1994) and Gries (2001) claim that there is a tendency towards the continuous order as NPs increase in length. Tables 3, 4, 5 and 6 represent the number of words in NPs according to each particle; $\mathrm{C}$ stands for Continuous and D stands for Discontinuous.

Table 3. NP Length -1 Word

\begin{tabular}{|l|c|c|c|}
\hline & \multicolumn{3}{|c|}{ 1 word } \\
\hline Particles & C & D & Freq. \\
\hline Away & $3=75 \%$ & $1=25 \%$ & $4=100 \%$ \\
\hline Off & $51=92.8 \%$ & $4=7.2 \%$ & $55=100 \%$ \\
\hline Out & $70=92 \%$ & $6=8 \%$ & $76=100 \%$ \\
\hline Total & $\mathbf{1 2 4}=\mathbf{1 0 0 \%}$ & $\mathbf{1 1 = 1 0 0 \%}$ & \\
\hline
\end{tabular}

Source: The British National Corpus (BNC).

NPs that are one-word length and that do not include pronouns are more frequent in the continuous order (75\%: away; 92.8\%: off and 92\%: out) in spite of some occurrences in the discontinuous form, as exemplified in (15).

15. Industry preparing for the Government legislation is luring inspectors away faster than they can be recruited to enforce it.

When NPs were formed by only one word, they were plural nouns, such as inspectors. Contrary to the claim that particles are more likely to be adjacent to verbs when NPs increase in size, there is actually more variety between continuous and discontinuous constructions when it comes to NPs with two words used with off. 
Table 4. NP Length - 2 Words

\begin{tabular}{|l|c|c|c|}
\hline & \multicolumn{3}{|c|}{ 2 words } \\
\hline Particles & C & D & Freq. \\
\hline Away & $20=77 \%$ & $6=23 \%$ & $26=100 \%$ \\
\hline Off & $75=88 \%$ & $10=12 \%$ & $85=100 \%$ \\
\hline Out & $203=95 \%$ & $11=5 \%$ & $214=100 \%$ \\
\hline Total & $\mathbf{2 9 8}=\mathbf{1 0 0 \%}$ & $\mathbf{2 7}=\mathbf{1 0 0 \%}$ & \\
\hline
\end{tabular}

Source: The British National Corpus (BNC).

As far as the use of off is concerned, NPs with one word are discontinuous to their verbs in $7.2 \%$ of the occurrences, but $12 \%$ when 2 words are used. In V NP P constructions, NPs were formed by definite articles (the) and possessive pronouns ( $m y$, his and her). There were instances of modifiers such as adjectives, but they were not as frequent as articles and possessives. Even so, the continuous order is by far the most frequent ordering with all three particles. Tables 5 and 6 show interesting results, as variety starts to decrease again.

Table 5. NP Length -3 Words

\begin{tabular}{|l|c|c|c|}
\hline & \multicolumn{3}{|c|}{ 3 words } \\
\hline Particles & C & D & Freq. \\
\hline Away & $16=100 \%$ & $\varnothing$ & $16=100 \%$ \\
\hline Off & $36=100 \%$ & $\varnothing$ & $36=100 \%$ \\
\hline Out & $128=98.0 \%$ & $3=2 \%$ & $131=100 \%$ \\
\hline
\end{tabular}

Source: The British National Corpus (BNC).

Table 6. NP Length - 4 Words or More

\begin{tabular}{|l|c|c|c|}
\hline & \multicolumn{3}{|c|}{ 4 or more words } \\
\hline Particles & C & D & Freq. \\
\hline Away & $22=100 \%$ & $\varnothing$ & $22=100 \%$ \\
\hline Off & $52=100 \%$ & $\varnothing$ & $52=100 \%$ \\
\hline Out & $112=98.0 \%$ & $2=2 \%$ & $114=100 \%$ \\
\hline
\end{tabular}

Source: The British National Corpus (BNC).

In relation to particles away and off, the continuous order is used in $100 \%$ of the cases when NPs have three or more words. Even though this result is not exactly the same for out, particles are still adjacent to verbs in the majority of the cases $(98 \%)$. According to Tables 5 and 6, there were few tokens in which NPs included three or more words in the discontinuous order, and they all refer to particle out. Data from (16) to (20) represent such results.

16. The agreement to let the original refugees out had been on a once-only basis.

17. The company is also hoping to exploit the link to pump its own programmes out to the thousands of mainstream and cable stations in America.

18. Many's the time I have found myself torn between throwing people like him out and putting up with them for the sake of the cash-till. 
19. It was a visit to Peking by the Soviet president in May that helped to bring more than a million demonstrators out on to the streets on two successive days.

20. When the lizards were dropped, they held their legs and tails out like good free-fall parachutists.

As can be seen from the instances above, (16) and (19) include TPVs whose NPs start with articles (the original refugees) or article + a quantitative construction (more than a million demonstrators). The NPs in (17) and (20) start with pronouns, which makes (18) the only exception since it has a full NP, starting with a noun: people like him.

The results displayed so far show that the continuous order is preferable over the discontinuous one in relation to all three particles, in spite of the number of words that form the NPs. Variety is higher when NPs are two-word in length, with nouns modified by articles and possessive pronouns at their most.

The expectation regarding the results is that one word ordering is favoured over the other and that some TPVs can be considered as chunks due to the lack of variability in relation to particle placement. Bearing this in mind, I decided to evaluate each construct in order to analyse type frequency, that is, the variety of verbs found in the sample so as to verify if certain verbs are more used with a specific particle and in a specific order. The purpose of this analysis was to search for constructions that formed sequential and crystallized units due to their token frequency. In the next section, graphics represent the most frequent verbs with particles away, off and out in addition to a brief analysis of their use, either continuously or discontinuously.

Particle "away"

For particle away, the verb throw is the most frequent one and it is used exclusively in the continuous order ( 9 tokens). Also recurrent in the sample is the verb take, which was used eight times in the continuous order and two in the discontinuous order. The verb sweep has particle away always adjacent to it in the seven times it was used. The verbs give, clear and carry had less significant results, but directly precede the particle as well. Finally, many verbs were also in the continuous order, but used only once: blow, blast, brush, cart, chase, drive, eat, explain, gamble, keep, lure, leg, put, scare, send, strip, shoo, turn, while, wipe and wash. Discontinuously, the following verbs had only one token with away: wash, get, lure, eat and brush. Figure 1 represents the most recurrent verbs from the 28 different ones that aligned with away in the corpus.

It is worth pointing out that throw is not only the most frequent verb in the sample, but it is also found only in the continuous order and with NPs that varied from two to five words, as demonstrated in examples (21) and (22):

21. We have all heard stories about GPs alleged to have given patients short shrift with remarks like: "Throw away your crutches. It's your head that needs them, not your legs". 
22. Would it be possible to re-use old programs written for conventional machines, or would users have to start from scratch and throw away their existing investment in software?

Figure 1. Frequency of Verbs Used With Particle "Away"

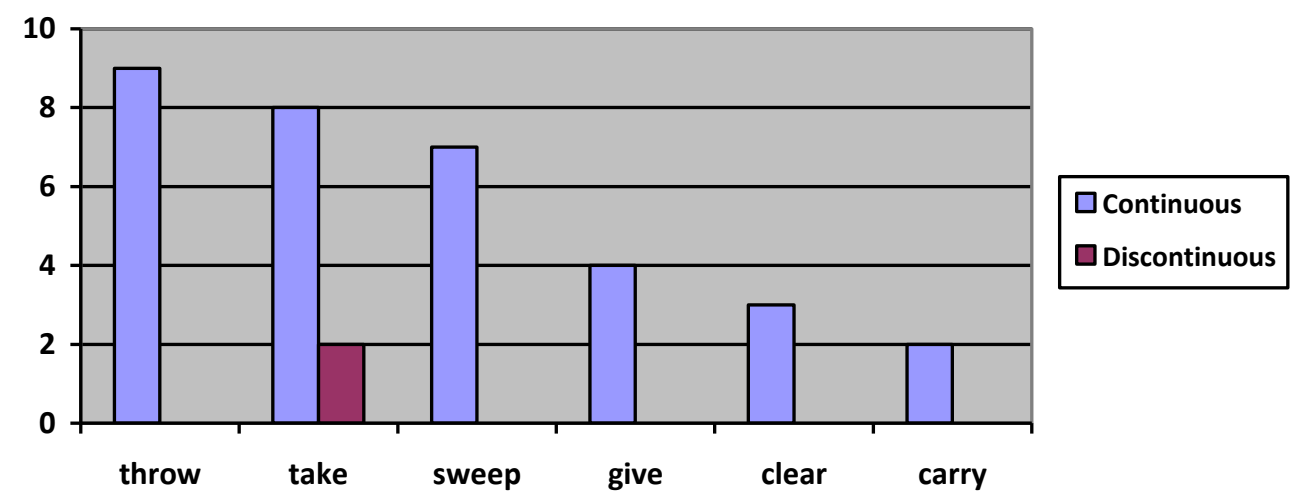

Source: The British National Corpus (BNC).

NPs varied semantically and in size, but throw and away behaved in the same way within all tokens in terms of $\mathrm{P}$ placement, which is evidence that a sequential unit is formed between the two constituents, whether NPs were short or long in size. Such attachment was not observed with the verb take, for instance, which had some instances of $\mathrm{P}$ being discontinuous to $\mathrm{V}$.

Particle "off"

As far as the particle off is concerned, there were more verbs in the sample in comparison with away. Figure 2 represents the relation between the 13 most frequent verbs in a total of 82 collected, showing that type frequency of constructions with off is higher than the ones with particle away.

Figure 2. Frequency of Verbs Used With Particle "Off"

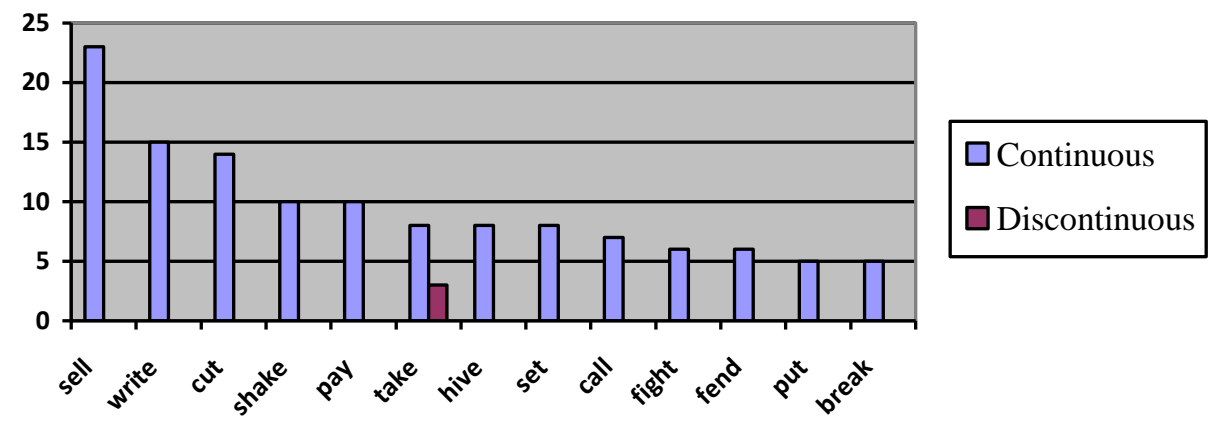

Source: The British National Corpus (BNC). 
Figure 2 shows that off is aligned with a wide range of verbs and mostly adjacent to them, which represents a high type frequency. The verb take is an exception to the list, since it is found in the discontinuous order as well.

In respect to the particle in question, the verb sell is the most recurrent one, found in 23 instances of the sample and in V P NP order. Write and cut are also recurrent and are part of 15 and 14 constructions, respectively. In addition to the verbs represented in Figure 2, a list of others were used only once: back, beat, bite, blaze, block, boil, burn, bum, carry, cast, cream, dust, finish, fire, get, head, hear, hold, hope, hop, keep, kick, kill, land, leap, let, lid, meet, pass, pack, pull, push, pare, pick, rattle, roll, round, rust, score, seal, see, send, shave, shrug, stave, star, strip, switch, scare, scream, slip, spark, start, turn, tick, ward and wipe. Although very rarely, off was separated from verbs other than take: get, hear, meet, pass, put, scare, scream, send, slip, throw and write.

As for the NPs' characteristics, they varied from one to seven words in size. This result shows that the number of words that are part of constructions $\mathrm{V}+$ off + NP is superior to the number of words found in the sample with particle away. (23) is an example of an NP with one word while (24) has seven words:

23. Mr. Ridley would not be drawn on the thorny issue of rail privatisation other then to say that investment was urgently needed in Britain's rail and road links. If this was stimulated by selling off $\underline{B R}$, so much the better.

24. Racal proved this conclusively when it announced last spring that it was planning to sell off part of the Vodafone mobile telecoms operation.

Figure 2 confirms the same ordering as Figure 1, i.e. verbs and particles (away and off so far) are constantly joined together and particle is separable from $\mathrm{V}$ in few instances of the sample.

Particle "out"

The type frequency as far as out is concerned is higher when compared to away and off. Various verbs were present only once in the continuous constructions considering the entire sample, adding up to a total of 96 different verbs. The following list includes the verbs found in the continuous order, from the most frequent to the least frequent one: a) verbs that were present more than once in constructions: carry, rule, take, work, bring, wipe, set, point, pay, buy, read, seek, single, weed, spell, send, hand, throw, hold, cut, hammer, iron, pick, pull, clear, find, drive, put and call; b) verbs that appeared only once when preceding particle out: act, bail, balance, bawl, bear, bellow, belt, bomb, bulk, cancel, catch, check, churn, clean, close, come, contract, cry, croon, cross, dole, dish, drown, dug, edge, farm, figure, fill, fish, fit, flat, flesh, freeze, get, give, ground, help, hand, knock, lay, kit, leave, level, make, map, mark, move, opt, phase and play.

The variable list of verbs previously mentioned shows that out is more productive in comparison with away and off. Taking into account the high type 
frequency of continuous constructions with out, Figure 3 focuses on the verbs which were more recurrent within the data found, which includes verbs carry, rule, take, work, bring, wipe and set.

Figure 3. Frequent Verbs Used With Particle "Out"

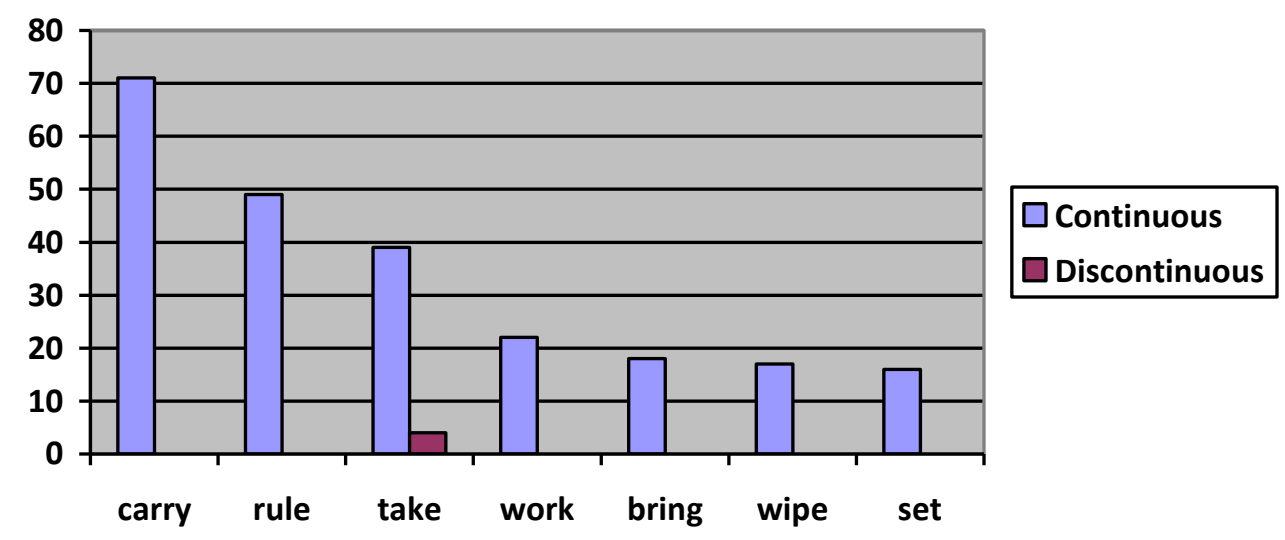

Source: The British National Corpus (BNC).

It is worth pointing out that the occurrence of carry was highly significant in the sample, appearing in 71 different tokens and exclusively in the continuous order. There is no other data showing verb and particle separated by NPs other than clitics.

As far as NPs are concerned, they also varied in terms of number of words, going from one up to five words, but all of them seem to bear a semantic resemblance to one another. Observe (25), (26) and (27) for this matter.

25. Since then NMW has carried out a heavy rationalisation programme.

26. The group (...) condemns the Government for failing to carry out research into its effectiveness.

27. Dr. Steven Clarke, (...) who carried out the survey, says the results highlight wide disparity in the use of thermometers.

Through the analysis of the sample as a whole, the NPs that followed the chunk carry out were all semantically related to the examples above. In addition to programme, research and survey, I found the following NP heads in the written sample collected: plan( $(s)$, experiment( $(s)$, test $(s)$, processes, instructions, study and operations, amongst others. Considering that NPs bore a semantic similarity in this construction, I wonder if the type of NP is a motivation for the selection of such a chunk. It must be pointed out that text genre seem to be linked to these results, since the continuous order can be found in other contexts, such as magazines. The examples below show the verb carry and the particle out in a discontinuous construction, and NPs here differ in meaning in comparison to the ones attested in the continuous order. 
28. They had to carry a table out with them, which was set up the required.

29. If he gets in through a window, he can't carry your property out through the door.

The construct carry + a table + out and carry + your property + out seem to differ from the constructions of the news' sample considering the semantic difference between table/ property and the NPs exemplified in (25), (26) and (27). Also, the value of the constructions in (28) and (29) is more compositional than when out is continuous to $\mathrm{V}$, i.e., meaning is built up through the sum of its parts: carry (to hold or support while moving ${ }^{7}$ ) is added to out (in a direction away from the inside ${ }^{8}$ ) being separate by NPs like table or property, which are concrete and physically handled objects. When out is adjacent to carry, meaning is conveyed from both constituents together and not from the sum of its parts. Thus, the semantic characteristics of the NP are directly connected to the user's choice of using a continuous or a discontinuous construction. However, this supposition is based only on the sample used for this work. A wider variety of texts should be analyzed for the purpose of confirming this behavior with this sort of construction. In addition, a comparison with spoken data may serve to support this hypothesis and/or to show there is a difference between the two discourses when it comes to particle placement in TPVs.

\section{Conclusion}

In this paper, I investigated the possible motivations for variable particle positioning in transitive phrasal verbs following Bybee's claim that frequent repetition of two or more words together tend to affect accessibility (2010). The main hypothesis was that TPVs tend to follow one order due to the high token frequency of the construction; for that reason, some constructs have become chunks in spite of semantic compositionality. In the interest of collecting evidence to support this argument, a quantitative analysis took place so as to verify general TPVs' token frequency. Results reveal that the V P NP order is significantly favourable in relation to V NP P when particles away, off and out are used in written discourse and in accordance with the sample selected for this work. Considering the written sample examined for this paper and comparing both the continuous and discontinuous orders, particles are adjacent to verbs $89.7 \%$ of the times in relation to particle away, $98.1 \%$ in relation to particle off and $96.3 \%$ for particle out.

When part of continuous constructions, NPs varied in terms of grammatical categories, but had more limitations in discontinuous constructions. Most of the NPs included nouns modified by articles or possessive pronouns, and they were separated according to the number of words. $86.6 \%$ of the times NPs were formed by one word only in the continuous order, but variety increased when two words were used, contrary to previous claims that the continuous order is more likely to

\footnotetext{
7 "Carry". TheFreeDcitionary.com. Retrieved from goo.gl/3hbMto. [Accessed: 4 January 2016]

8 "Out". TheFreeDictionary.com. Retrieved from goo.gl/NXpA5q. [Accessed: 4 January 2016]
} 
take place as number of words increase. Only when NPs contained three words or more, particles were, again, more often adjacent to verbs: $99.3 \%$ of the times.

The construction throw + away was the most frequent one as regards to away, occurring nine times in the sample and exclusively in the continuous order. As for off, a wider range of verbs was used in comparison with away, which shows a difference of particle use in terms of type frequency. It is worth pointing out that the verb take is part of both continuous and discontinuous constructions regarding off and away, which reveals that variable particle positioning appears to be more frequent with take.

There were 68 tokens for particle away, 233 for particle off and 576 for particle out. A total of 877 transitive phrasal verbs were found in the sample, which makes out part of $65.7 \%$ of the tokens. The frequency of out in relation to all data was considerably larger than the other two particles, and not only was the number of tokens vaster, but also the range of verbs that aligned with out. In other words, constructions with out have a higher type frequency than the ones found with away and off. Also, the tendency to the usage of the continuous order with most of the verbs and specially with the verb carry shows that variable particle positioning seems to apply more to verbs such as take (which occurred with all three particles and in both orders) than with carry.

In addition to having a significantly higher token frequency in relation to the other verbs and particles in the study, the construction carry out remained exclusively in the continuous order, showing a strong entrenchment and high token frequency between verb and particle. Thus $\mathrm{V}$ and $\mathrm{P}$ seem to be packaged and stored together rather than being separately selected so as to form meaning, indicating an apparent process of chunking in TPVs. This link between verb and particle seem to be related to the NPs used, considering they were all semantically related. It was briefly shown that, when carry $+N P+$ out occur an ordering not found in this sample and only provided for the purposes of exemplifying such case -, NPs are semantically similar, but different from the ones used in the continuous order. This conclusion is based only on the observation of newspapers corpora, which reinforces the need for an analysis that covers other text genres so as to verify the nature of the NPs used with carry out. If NPs that are adjacent to $\mathrm{V}$ are semantically and/or pragmatically different from the ones used in the discontinuous order, this is an indication there are in fact two different constructions established based on the NPs characteristics.

\section{References}

Biber D, Conrad S, Leech G (2010) Student Grammar of Spoken and Written English (9 $^{\text {th }}$ edn.). England: Longman.

Bybee J (2010) Language, Usage and Cognition. Cambridge: Cambridge University Press.

Bybee J, Eddington D (2006) A usage-based approach to Spanish verbs of "becoming". Language 82(2): 323-355.

Claridge C (2000) Multi-word Verbs in Early Modern English: A Corpus-based Study. Amsterdam: Brill, Rodopi. 
Goldberg A (2016) Tuning in to the verb-particle construction in English. In L Nash, P Samvelian (Eds.), Approaches to Complex Predicates. Boston: Brill.

Gorlach M (2004) Phrasal Constructions and Resultativeness in English: A sign-oriented analysis. Amsterdam, The Netherlands: John Benjamins.

Gries ST (2001) A multifactorial analysis of syntactic variation: particle movement revisited. Journal of Quantitative Linguistics 8: 33-50.

Haddican B, Johnson D (2012) Effects on the Particle Verb Alternation across English Dialects. Working Papers in Linguistics, no. 18(2), University of Pennsylvania.

Hawkings J (1994) A Performance Theory of Order and Constituency. Cambridge: Cambridge University Press.

Kemmer S, Barlow M (2000) Introduction: Usage-Based Models of Language. In M Barlow, S Kemmer (Eds.), Usage-Based Models of Language (vii-xxviii). Stanford: Center for the Study of Language and Information.

Lohse B, Hawkins JA, Wasow T (2004) Domain minimization in English verb-particle constructions. Language 80(2): 238-261.

Quirk R, Greenbaum S, Leech G, Svartvik J (1985) A comprehensive grammar of the English language. London: Longman.

The British National Corpus, version 3 (BNC XML Edition) (2007) Distributed by Oxford University Computing Services on behalf of the BNC Consortium.

Traugott EC, Trousdale G (2013) Constructionalization and Constructional Change. Oxford: Oxford University Press. 
